\title{
Social Distancing Was Practiced to Aggravate the COVID-19 Pandemic
}

\author{
April 13, 2019
}

Jianqing Wu, Ph.D., J.D.

After the COVID-19 pandemic has spread rapidly for some time, I finally saw the hope that the pandemic would be brought under control. This change had been made at the cost of shutting down the national economy. It is just the start of the pandemic and we must think about the effectiveness of public health intervention measures. Several instances I have encountered convinced me that there are great room to improve in the future. I believe acknowledging problems in current measures is necessary to achieve better results in the future.

Social distancing requires that two persons in a public place to maintain at least 6 feet distance. This distance can prevent viral transmission through droplets or relatively large particles from an infected person to an uninfected person. Since coughed droplets and relatively large viral particles can travel only short distances, six feet distancing can stop such particles transfer from an infected person to a healthy person. However, this measure does not stop very small viral particles that are suspending in the air. Despite lack of consensus on such viral particles, this mechanism is implied in stories in China.

Social distancing is a practice intended to reduce the degree of viral exposure. A presumption is that it should not be practiced at the expense of customer health or increasing the amount of viral particles that customers are exposed to. However, three instances I have personally experienced can actually make customer sick and increase the degree of viral exposure.

In one instance, a store tried to limit the number of customers insider the store. It was a rainy day, the clerk required all customers to stand in a long line. Most people wore neither rain coat nor adequate clothing. After standing in icy-cold rain with strong wind for more than half an hour, many of them could get cold later. Temperature is the most important factor that affects the body's ability to fight the virus through a large number of known mechanisms. Based on existing knowledge, exposure to low temperature is the most effective way to temporarily cripple the body's defense ability. In this case, social distancing was practiced to make customers sick.

In a second instance, a store made some administrative changes to enforce social distancing practice. It pulled the clerks from check-out cash registers and assigned them to maintaining line order and disinfecting shopping carts, etc. The clerks maintained a very long line outside the store in a cold wind. One customer was allowed to enter only after a customer had left the store. Due to lack of cash registers, customers had to stand in two long lines inside the store. All customers wore masks, so that the rationale to keep 6 feet distance was no longer important. In China, proper use of masks was able to put down a large outbreak where human density is much higher. However, I found that this shopping instance was a perfect scenario of making more infection cases. The customers were first in a warning condition when they were in their own cars, they then were chilled for half an hour to one hour outside 
the store; and it is a sure way to cripple body's defense ability. Before the customers had a chance to restore their health conditions, they were forced to stay in the store for longer times to get more exposure to the virus. Based on my personal experience, the total time in this store was increased from 5 to 10 minutes that I normally spent to about two hours. Also the clerks made a new convention that no customer was allowed to load groceries on checkout bells before the current customer had left. So, the customer was kept at more than 6 feet distance. The store shut down the fast lanes and made old people to stay much longer. Because no mask is perfect, some virus must get into the respiratory tracks. The customers were exposed to more viral particles than they had to.

In the third instances, the problem is similar to the second one. The store had many clerks who stood by while the store had two long lines of customers for checkout. When I asked the store clerk to do something to reduce waiting times, the clerk said: they had to follow the social distancing requirement. I could not see any rule that whole store customers had to stand in long times while most clerks had little to do.

For my own personal reason, I was never worried about getting influenza in the past. Now, I have to worry about much more. Based on my knowledge, fluctuating temperature exposure cycles are most effective ways to cause the immune system suppressed. Based on my experience, the total shopping times are 2 to more times more than the times I spent before the 6 feet social distancing was enforced. It is really a nightmare.

The store clerks in those instances tried to enforce social distancing practice with good intention. However, the intervention becomes a terrible thing when it is practiced without exercising common sense and sound judgment. Since social distancing is intended to control viral transmission, it should not be practiced to make customers sick or force them to expose to the virus for longer times. The severity of viral exposure is directly proportional to time. 